\title{
ECG-Derived Respiration Using a Real-Time QRS Detector Based on Empirical Mode Decomposition
}

\author{
Christina Kozia \\ Mathematics Department \\ Aston University \\ Birmingham, UK \\ koziac@aston.ac.uk
}

\author{
Randa Herzallah \\ Mathematics Department \\ Aston University \\ Birmingham, UK \\ r.herzallah@aston.ac.uk
}

\author{
David Lowe \\ Mathematics Department \\ Aston University \\ Birmingham, UK \\ d.lowe@aston.ac.uk
}

\begin{abstract}
Respiration Rate (RR) is an important physiological indicator and plays a major role in health deterioration monitoring. Despite that, it has been neglected in hospital wards due to inadequate nursing skills and insufficient equipment. ECG signal, which is always monitored in a clinical setting, is modulated by respiration which renders it a highly enticing mean for the automatic RR estimation. In addition, accurate QRS detection is pivotal to RR estimation from the ECG signal. The investigation of QRS complexes is a continuing concern in ECG analysis because current methods are still inaccurate and miss heart beats. This paper presents a frequency domain RR estimation method which uses a novel real-time QRS detector based on Empirical Mode Decomposition (EMD). Another novelty of the proposed work stems from the RR estimation in the frequency domain as opposed to some of the current methods which rely on a time domain analysis. As will be shown later, the RR extraction in the frequency domain provides more accurate results compared to the time domain methods. Moreover, our novel QRS detector uses an adaptive threshold over a sliding window and differentiates large $Q$ - from R-peaks, facilitating a more accurate RR estimation. The performance of our methods was tested on real data from Capnobase dataset. An average mean absolute error of less than 0.5 breath per minute was achieved using our frequency domain method, compared to 6 breaths per minute of the time domain analysis. Moreover, our modified QRS detector shows comparable results to other published methods, achieving a detection rate over $99.80 \%$.

Index Terms-ECG-derived respiration, Frequency domain analysis, R-peak detection, Empirical Mode Decomposition (EMD), Local Signal Energy
\end{abstract}

\section{INTRODUCTION}

Many of the literature since the mid-1990s emphasises the importance of the respiration signal and its derivative, the Respiration Rate (RR). In [1] it was indicated that an RR higher than 27 breaths per minute is the most important predictor in failure of the heart to contract effectively in hospitals. In [2] the necessity of RR was investigated. It was claimed that $21 \%$ of hospitalised patients with an RR of 2529 breaths per minute assessed by a critical care outreach service died in hospital. An increase of the mortality rate has been reported for patients with higher RR [2]. It has been demonstrated that just over the half of unhealthy subjects suffering a serious event in the hospital wards has a RR greater than 24 breaths per minute and these subjects could have

This work is supported by Isasnys Lifecare. been identified as high risk up to 24 hours before the event with a specificity of over $95 \%$. The respiration signal can be recorded using the following methods: spirometry, pneumography, plethysmography, or capnography. The main disadvantage of these methods is that they use expensive equipment and make the patients' hospitalisation uncomfortable.

\section{A. Respiratory-Induced Modulation of ECG}

Research into extraction of respiration signal using the ECG has a long history. In [3] the use of the ECG in respiration acquisition was suggested by pointing out that a normal respiration cycle affects the heart rate, which causes sinus arrhythmia. More recently, it was observed that the ECG signal is affected by events occurring during the breathing process [4]. A change of heart-to-electrode distances is observed during the thoracic expansion which modulates the QRS morphology. Furthermore, a variation of amplitude, frequency, and phase is observed because the ECG is affected by changes in thoracic impedance as air fills spaces in lungs.

\section{B. ECG-Derived Respiration Methods}

The correlation between the RR and the ECG signal leads us to RR estimation through ECG. ECG signal is monitored routinely in a hospital setting, it is non-invasive, inexpensive, and safe. In recent years there has been an increasing amount of literature on ECG-derived (EDR) respiration methods [4] [5]. The alternation of QRS morphology facilitates the respiration signal extraction because it is related to the breathing cycle.

In [5] an EDR method which is based on peak-to-trough QRS amplitude was suggested. A single lead ECG signal is given as input to the algorithm. The R-peaks of the signal are detected and the peak-to-trough amplitude is measured. As soon as all R-peak amplitudes are measured, a cubic spline interpolation is attempted followed by a filtering stage in order to extract the respiration signal. In [4] the so-called Respiratory Sinus Arrhythmia (RSA) derived respiration method was proposed. The latter uses the R-peak time locations in order to compute the R-R intervals. The instantaneous heart rate (IHR) values are then computed. IHR is in fact the inverse of $\mathrm{R}-\mathrm{R}$ intervals. After successful computation of the IHR values, 
the latter are interpolated using cubic splines, followed by a filtering stage in order to derive the respiration signal.

After successful extraction of the respiration signal, the RR estimation is attempted, using a time domain analysis method. In [6] it is suggested to use a basic peak detector in order to extract the peaks in the respiration signal, along with their time locations. The instantaneous respiration rate (IRR) is evaluated from the time intervals of consecutive peaks in the respiration signal. As soon as the IRR values are computed, they are converted to breaths per minute to get the RR.

\section{QRS Detection}

The QRS complex is the most distinguishable feature of the ECG signal and comprises valuable information which facilitates the estimation of the RR [3] and Heart Rate Variability [7]. R-peak detection complexity results from signal contamination due to noise and the QRS morphological variability due to respiration. Furthermore, the complexity of the QRS identification stems from the difficulty of differentiating Rpeaks from large $\mathrm{P}$ or $\mathrm{T}$ peaks [8].

A great deal of previous research has focused on the QRS detection using a variety of approaches from derivative based methods [8] [9] to neural networks [10] [11] methods. The majority of the algorithms first pre-process the signal, in order to enhance the QRS complex and reduce noise, and then apply a set of thresholds for the R-peak identification. Signal differentiation [8] [9] and Hilbert transform [12] [13] have been proposed as pre-processing methods for QRS amplification. Most recently, Empirical Mode Decomposition (EMD) [14] has been proven to be an effective QRS pre-processor [15] [16] [17]. The R-peaks are finally located by a set of thresholds such as: estimators of signal and noise level like the median [8] or the Root Mean Square (rms) [12], the heart refractory period (200 miliseconds) [18], or thresholds based on the maximum amplitude of the signal [16] [17]. A major weakness of the detectors being proposed in [15], [16] and [17] is that the threshold is derived from the full length ECG. Ectopic Rpeaks can make the threshold high which results in the failure to detect lower R-peaks. Our QRS detector provides a solution to the latter by dividing the signal into segments. An additional drawback of [15], [16] and [17], which emerges from the use of the full length ECG for the threshold computation, is that these methods cannot be implemented on-line. In contrast, we propose a real-time QRS detector which only makes use of the most recent history of the ECG signal.

\section{Empirical Mode Decomposition}

EMD decomposes the signal, $x(t)$, into a series of narrowband signals, $c_{i}(t)$, which are called IMFs, and fulfill special conditions. An oscillatory mode of the signal is an IMF exclusively under the conditions that: first, in the whole dataset, the number of zero-crossings and the number of extrema are either equal or differ at most by one; and second, at any point, the mean value of the maximum and the minimum envelope is zero. The key advantage of EMD is that it is a data-driven analysis method. In each iteration the algorithm needs to decide if the $i$-th component, $h_{i}(t)$, extracted from the data, is an IMF based on the conditions mentioned above. In order to achieve this, the EMD method uses a systematic way which is called the sifting process and is described as follows: for a given signal $x(t)$, the extrema points are first identified, followed by approximation of the upper, $\widehat{r}(t)$, and lower, $\underline{r}(t)$, envelopes of the signal through a cubic spline interpolation. The mean is then obtained, and the $i$-th component, $h_{i}(t)$, is computed as the difference between the signal and the mean. The sifting process has to be repeated as many times as required to reduce the extracted signal to an IMF. For our implementation in order to terminate the EMD algorithm, the number of zero-crossings and the number of extrema are checked for equality or whether they differ at most by one. If the final residue, $r_{N}(t)$, is obtained as a monotonic function, the sifting process is stopped, $c_{i}=h_{i}$, and the signal, $x(t)$, can be written as follows:

$$
x(t)=\sum_{i=1}^{N} c_{i}(t)+r_{N}(t),
$$

where $N$ is the total number of the extracted IMFs.

\section{E. Overview of the Proposed EDR method}

Our EDR method first locates the QRS complexes. For each QRS complex the peak-to-baseline amplitude is computed and outliers due to noise and artifact are discarded. As soon as the peak-to-baseline values are evaluated, the sequence is upsampled using a cubic spline, followed by a filtering stage in order to derive the respiration signal. The latter is then divided into one minute windows in order to estimate the RR. For each window the Fourier transform of the respiration signal is computed. The next step requires the identification of the frequency that corresponds to the most dominant peak in the frequency spectrum, which is assumed to be the respiration rate in the current window. The novelty of our EDR method lies on the fact that the RR estimation takes place in the frequency domain, whereas time domain analysis is sensitive to dicrotic notches, which makes RR estimation inaccurate. Moreover, our frequency analysis approach can be implemented in real-time as the estimation requires windows of one minute duration.

Our proposed QRS detector overcomes the aforementioned problems in the current state-of-the-art EMD methods by introducing an adaptive threshold which is calculated from the local energy of the reconstructed ECG signal from the EMD. The signal is first pre-processed by a bandpass filter and EMD in order to eliminate noise, baseline wander and enhance the QRS complexes. Our R-peak detector has a number of attractive features, compared with the existing results on the topic. Firstly, our adaptive threshold, derived from the local signal energy of a prespecified number of most recent segments, prevents the failed detection of small R-peaks. Secondly, the present approach explores the differentiation of R-peaks from large $\mathrm{Q}$ peaks in the absolute value of the signal, by using the first derivative of the ECG signal. Thirdly, our method 
proposes an on-line algorithm with a detection delay equal to the duration of the segment and a low memory usage.

\section{Methods}

\section{A. Proposed Frequency Domain ECG-Derived Respiration}

The proposed RR estimation method is based on the fact that the most dominant peak of the respiration frequency spectrum corresponds to the RR. In order to derive the respiration signal, the R-peaks are located using our proposed QRS detector (Section II-C). In contrast to existing EDR methods [4] [5] the peak-to-baseline amplitude (PBA) for each QRS complex is computed because the ECG signal is first filtered in order to remove baseline wander and noise during the R-peak detection (Fig. 1, Plot 1). In order to increase the accuracy of the RR estimation, outliers are discarded to minimise the presence of false positives due to R-peak detection. Then the PBA values are upsampled in order to derive the EDR waveform. The latter is then filtered within reasonable respiration frequencies in order to extract the final respiration signal (Fig. 1, Plot 2). The next step is the frequency domain analysis of the respiration signal where a Fourier transform is applied to one minute windows of the respiration in order to estimate the RR (Fig. 2). To summarise, the proposed EDR method is as follows:

1. Extract the QRS complexes from a single-lead ECG signal,

2. For each QRS complex compute the peak-to-baseline amplitude (PBA),

3. Discard outliers by restricting PBA values to remain within the range of \pm 2 SDs from the mean value,

4. Upsample the remaining PBA values at 8 Hertz using cubic spline interpolation,

5. Filter the output of step 4 within reasonable respiration frequencies $(0.0666-0.5 \mathrm{Hertz})$ to get the respiration signal,

6. Divide the respiration signal into one minute windows,

7. For each window compute the Fourier transform of the respiration signal,

8. Locate the frequency that corresponds to the most dominant peak in the frequency spectrum,

9. Convert frequency to breaths per minute to get the respiration rate.

\section{B. Time Domain ECG-Derived Respiration}

One popular approach for the RR estimation is based on the assumption that the time interval between two peaks in the respiration signal, is the interval between two breaths [6]. In other words, the time domain analysis of the respiration attempts to find the sampling interval between the peaks of the respiration signal and convert it to breaths per minute (Fig. 3). To summarise, the time domain analysis for the RR estimation is as follows:

1. Derive the respiration signal as proposed in Section II-A,

2. Extract the time locations of the peaks of the respiration signal, that is $t_{1}, t_{2}, \ldots, t_{n}$, where $n$ is the total number of peaks in the respiration signal,

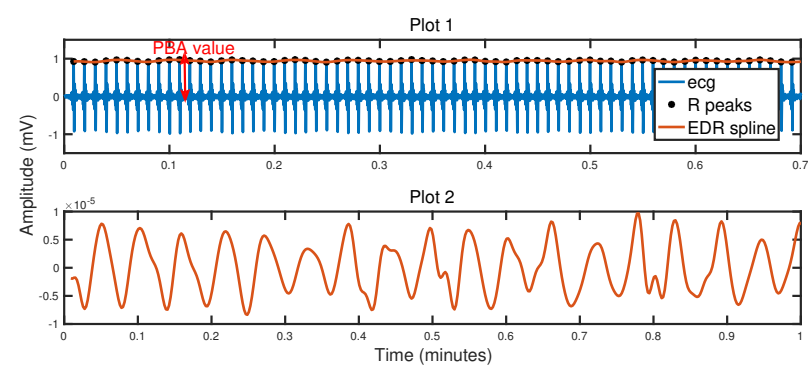

Fig. 1. Plot 1 shows our proposed EDR method for a small part (0.7 minutes) of an ECG. R-peaks are represented by black dots. The output of the EDR interpolation is represented in a red curve. Plot 2 shows the derived respiration signal for a one minute window corresponding to the ECG of Plot 1.

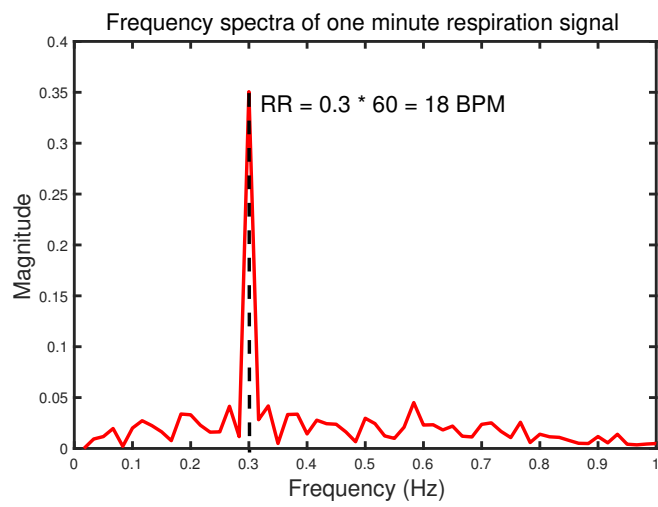

Fig. 2. The frequency spectrum of the one minute window respiration signal of Fig. 1, Plot 2. The dominant peak is located (black dashed line) and it is converted to breaths per minute (bpm).

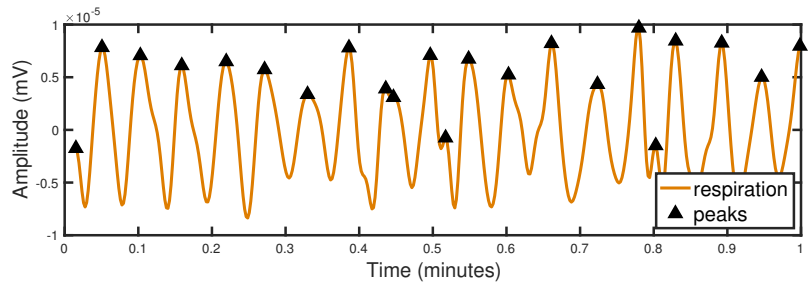

Fig. 3. Time domain analysis for one minute window. The peaks used in RR estimation are represented by black triangles.

3. Compute the IRR values,

$$
I R R_{i}=60 \cdot \frac{1}{t_{i+1}-t_{i}},
$$

where $i=1, \ldots, n-1$,

4. For every one minute window, take the average of the IRR values within that window in order to get the RR in breaths per minute.

\section{Proposed R-peak Detection}

The main assumption of the proposed R-peak detector is that the QRS complex can be amplified by reconstructing the signal from the first three IMFs of the EMD. This assumption, which will be discussed in section III, was verified on all the tested recordings. The signal is first pre-processed by a 
bandpass filter and EMD to reduce baseline wander, noise and enhance the QRS complexes. Then the reconstructed signal is divided into a number of segments. The envelope of the maxima of each segment is approximated and followed by the computation of the local signal energy and an averaging step for the evaluation of the threshold. The final R-peak decision requires a gradient-based and a refractory period checks to prevent false detection. The chosen segment duration provides an adequate number of QRS complexes and depends on the sampling frequency. Moreover, for the averaging step the eight most recent segments were used. The number of segments to be averaged is a prespecified parameter which can be decided based on the clinical condition of the patient and whether it is expected that their ECG signal is going to be less or highly variant. However, it is recommended that just the most recent history of the ECG vital signs are kept. To summarise, the proposed QRS complex detection algorithm is as follows:

\section{Pre-processing Stage}

1. The raw signal, $x(t)$, is first filtered with a band-pass filter, whose coefficients were designed using a KaiserBessel window [19]. The band-stop frequencies were set at 8 and 20 Hertz [20] in order to amplify the QRS complex, eliminate noise and reduce the number of IMFs. The output of the filter is denoted as $x_{f}(t)$,

2. The EMD method is applied on $x_{f}(t)$ to extract the IMFs, $c_{1}(t) \ldots c_{N}(t)$, where $N$ is the total number of the extracted IMFs,

3. The signal is reconstructed by adding the first three IMFs,

$$
x_{r}(t)=\sum_{i=1}^{3} c_{i}(t),
$$

where the number of IMFs is experimentally selected and it will be discussed later,

4. Then, the absolute value of the reconstructed signal is computed, that is $a(t)=\left|x_{r}(t)\right|$. This makes all data points positive and implements a linear amplification of the reconstructed signal emphasising the higher frequencies.

\section{Decision Stage}

5. In order to increase the efficiency of the algorithm, we divide $a(t)$ into $k$ segments of 3 seconds duration, that is $k=$ (total number of samples) $/(3 * f s)$. The starting point of the $k$-th segment should match the last $\mathrm{R}$ peak located in the $k-1$ segment in order to increase the accuracy,

6. Compute the envelope of the maxima, $\hat{a}_{k}(t)$, of $a_{k}(t)$ for each segment $k$ through a cubic spline interpolation of the local maxima,

7. Compute the local signal energy for each segment as,

$$
R M S_{k}=\sqrt{\frac{1}{M} \sum_{t=1}^{M}\left[\hat{a}_{k}(t)\right]^{2}},
$$

where $k$ is the current segment and $M$ is the number of samples in the segment, that is $M=3 * f s$,
8. The threshold of the $k$-th segment is set to be the mean of the most recent eight $R M S_{k}$ values,

$$
T_{k}=\frac{1}{8} \sum_{j=k-7}^{k} R M S_{j},
$$

9. The peaks, which exceed threshold $T_{k}$ in the absolute sequence $a_{k}(t)$, are classified as candidate peaks.

10. In order to segregate large $\mathrm{Q}$ peaks from $\mathrm{R}$ peaks, we compute the first derivative of $x_{r}(t)$. Peaks with a negative derivative will be investigated further at the refractory period check given next,

11. Apply a refractory period check. When the R-R interval of two adjacent peaks is less than 200 milliseconds, keep the peak with the maximum amplitude.

\section{RESUlTS AND Discussion}

\section{A. ECG and RR Reference Data}

For the evaluation of our proposed methods, 20 children recordings (median age: 9.1, range: 1-16 years) and 5 adult recordings (median age: 46.2, range: 37-64 years) were used from the Capnobase dataset [21]. The database contains ECG, pulse oximetry and capnography recordings of 8 minute duration acquired during elective surgery and routine anaesthesia, sampled at 300 Hertz. Moreover, all recordings under study provide annotated R-peaks and reference RR.

\section{B. ECG-Derived Respiration}

For all the recordings the results are shown in Table I. In order to evaluate the performance of our proposed EDR method, the Mean Absolute Error (MAE) in breaths per minute (bpm) was calculated as follows:

$$
M A E=\frac{1}{N} \sum_{i=1}^{N}\left|R R_{r_{e f}}-R R_{e s t_{i}}\right|,
$$

where $R R_{\text {ref }}$ is the reference RR, $R R_{e s t}$ is the estimated RR, and $N$ is the total number of one minute windows.

As can be seen from Table I our EDR method shows a promising performance for most of the recordings, achieving an average MAE of 0.4150 breaths per minute. Fig. 4 shows the respiration signal obtained from our method for recording 0009. Each plot corresponds to one minute window. As soon as the respiration signal is ready, our method analyses the latter in the frequency domain in order to estimate the RR. Fig. 5 shows the frequency analysis of the respiration signal of recording 0009. As we have already mentioned, the ECG signals duration, from Capnobase dataset, is 8 minutes, thus we expect to see the frequency spectra of 8 one minute windows. Plots 1 to 8 of Fig. 5 show the frequency domain analysis for the full length respiration signal, along with the reference RR. It can be seen that each window contains a dominant peak which corresponds to the RR.

The largest MAE, which was obtained for recording 0032, is about 2.6240 breaths per minute. As can be seen in Fig. 6, the respiration signal shows smaller oscillations which can overlap the actual respiration, and it does not have a smooth shape as 
TABLE I

ECG-Derived Respiration Evaluation Performance

\begin{tabular}{|c|c|c|}
\hline \multicolumn{2}{|c|}{ Frequency Domain Analysis } & Time Domain Analysis \\
\hline Children Recordings & MAE (bpm) & MAE (bpm) \\
\hline 0009 & 0.3716 & 0.7478 \\
0015 & 0.2086 & 0.0710 \\
0016 & 0.0048 & 11.2462 \\
0018 & 1.2627 & 9.0078 \\
0023 & 0.2624 & 0.5929 \\
0032 & 2.6240 & 13.1101 \\
0035 & 2.0175 & 1.5300 \\
0038 & 0.6886 & 0.5697 \\
0103 & 0.0079 & 0.0873 \\
0104 & 0.0049 & 3.9799 \\
0105 & 0.0476 & 6.4162 \\
0121 & 0.0069 & 11.2100 \\
0122 & 0.0070 & 10.6559 \\
0125 & 0.3194 & 3.0763 \\
0127 & 0.3776 & 0.8477 \\
0128 & 0.4403 & 13.8068 \\
0134 & 0.0062 & 17.0908 \\
0142 & 0.5118 & 4.2626 \\
0147 & 0.0043 & 13.2784 \\
0148 & 0.0051 & 11.4484 \\
\hline Adult Recordings & & 8.1826 \\
0311 & 0.0263 & 13.7058 \\
0312 & 0.6346 & 7.5415 \\
0313 & 0.0110 & 0.5884 \\
0322 & 0.5099 & 14.1616 \\
0325 & 0.0150 & $\mathbf{6 . 6 9 8 7}$ \\
\hline Average & $\mathbf{0 . 4 1 5 0}$ & \\
\hline \hline & & \\
\hline
\end{tabular}
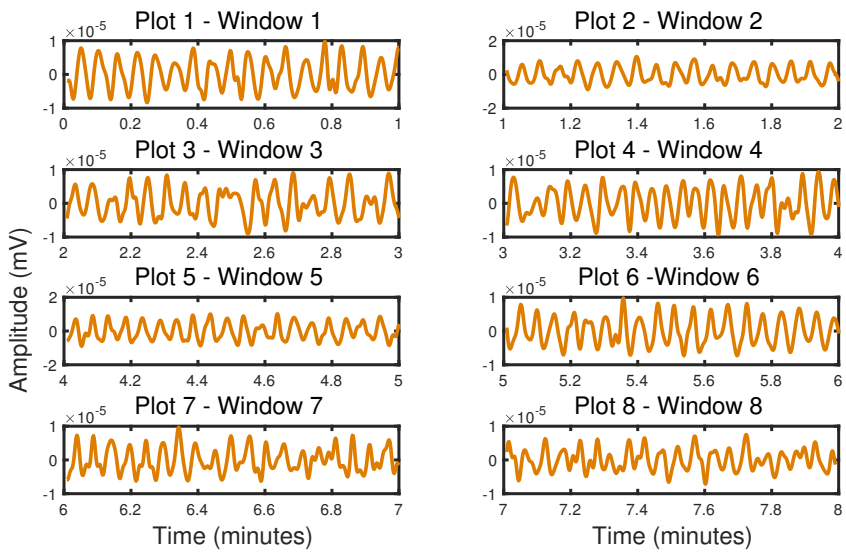

$\times 10^{-5} \quad$ Plot 6 -Window 6

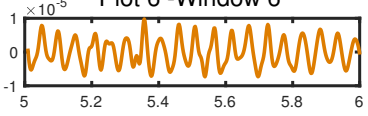

$\times 10^{-5} \quad$ Plot 8 - Window 8

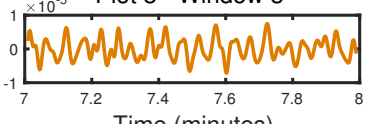

Time (minutes)

Fig. 4. The respiration signal obtained from our EDR method for recording 0009. Plots 1 to 8 correspond to the 8 one minute windows obtained from our algorithm.

the respiration of recording 0009 (Fig. 4). Our observation becomes more clear from the frequency domain analysis of the respiration signal. Fig. 7 shows the frequency spectrum of each one minute window for the respiration signal of recording 0032. What stands out in Fig. 7 is that there are several peaks close to the dominant, which means that there are overlapping frequencies, thus we cannot obtain the correct RR for all the windows.

In order to compare our RR estimation results, we have implemented the time domain analysis of the respiration signal
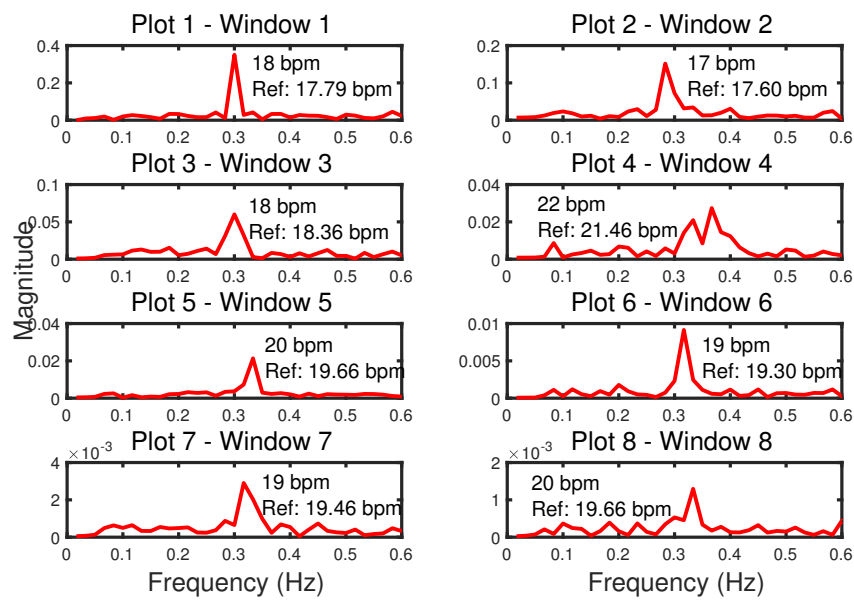

Fig. 5. Frequency domain analysis of the respiration signal obtained from our EDR method for recording 0009. Plots 1 to 8 show the frequency spectra of each one minute window, along with the reference RR.
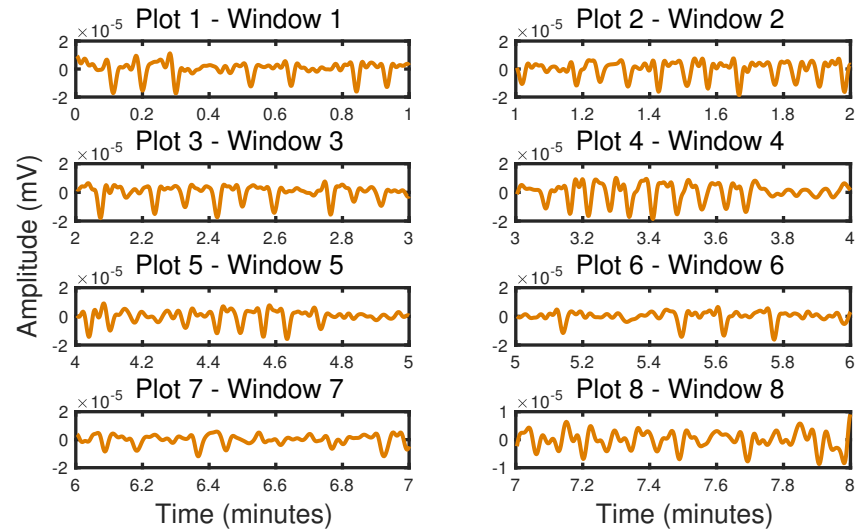

Fig. 6. The respiration signal obtained from our EDR method for recording 0032. Plots 1 to 8 correspond to the 8 one minute windows obtained from our algorithm.

described in Section II-B [6]. The results obtained from this approach are shown in Table I. It can be observed that the MAE obtained from the time domain analysis is high about 6 breaths per minute. The main drawback of this method is that dicrotic notches are also detected as peaks in the respiration signal. Fig. 8 shows the time domain analysis for the recording 0134. As can be seen a large number of dicrotic notches are detected as peaks, making the RR estimation inaccurate.

A major advantage of our proposed EDR method is that it shows promising results for both real children and adult data, without changing any parameters during the experiments. Moreover, as can be seen from Table I our proposed method outperforms the proposed time domain analysis, achieving an average MAE of 0.4150 breaths per minute compared to 6.6987 breaths per minute [6]. Furthermore, the practical advantage of this method is that it can be implemented on-line with an estimation delay equal to one minute, because the 

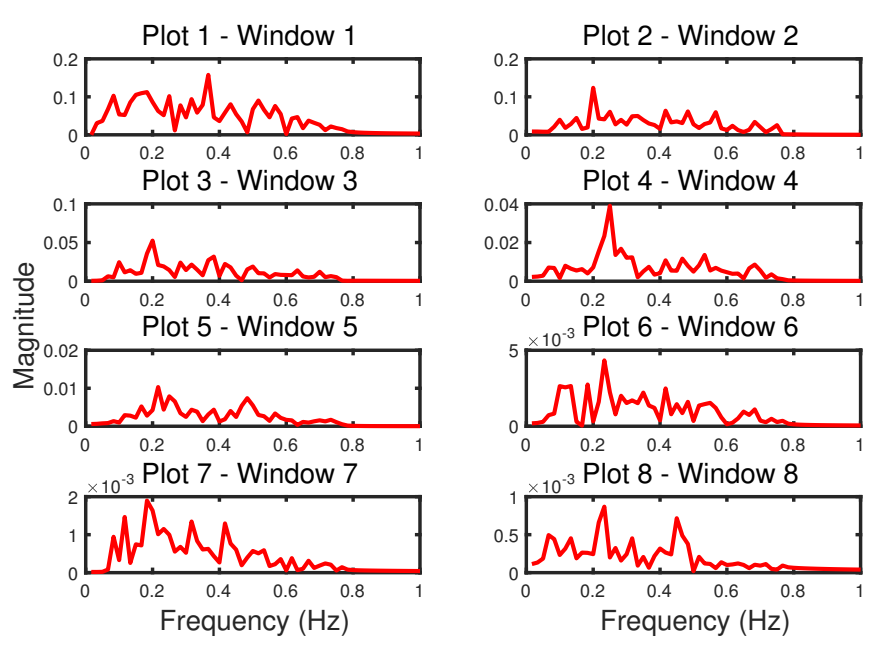

Fig. 7. Frequency domain analysis of the respiration signal obtained from our EDR method for recording 0032. Plots 1 to 8 show the frequency spectra of each one minute window.
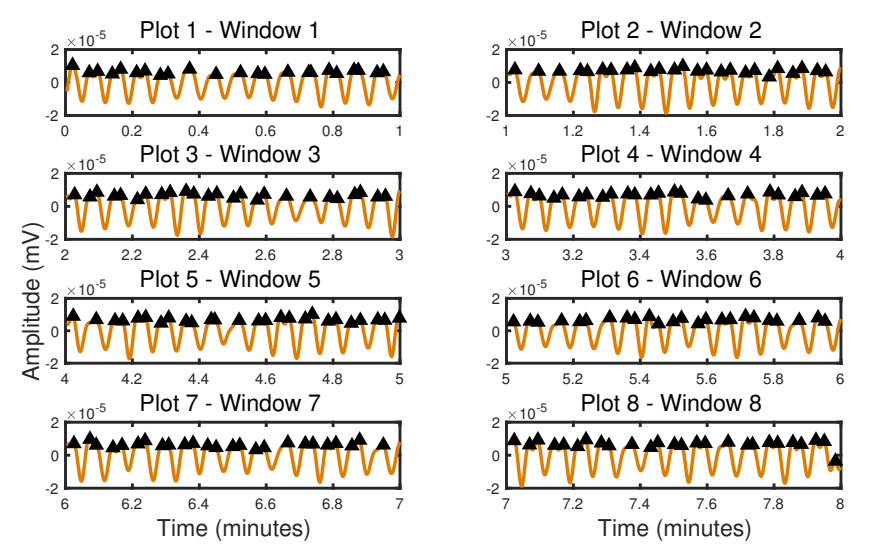

Fig. 8. Time domain analysis of the respiration signal obtained from our EDR method for recording 0134. Plots 1 to 8 correspond to the 8 one minute windows. The detected peaks, which are used in time domain analysis, are represented by black triangles.

frequency domain analysis of the respiration signal requires windows of one minute. The reduction of the duration of the estimation delay will be part of our future work.

\section{R-peak Detection}

The proposed QRS detector is based on the assumption that the range of the frequencies of the first three IMFs of the EMD correspond to the QRS complex which includes high frequencies in the range 3-40 Hertz [18]. Moreover, $\mathrm{P}$ and $\mathrm{T}$ wave frequencies are about 0.7-10 Hertz [18]. Therefore in order to amplify QRS complexes, the IMFs that correspond to $\mathrm{P}$ and $\mathrm{T}$ waves should be omitted. The validity of our assumption is shown below. Fig. 9 shows a filtered ECG signal and its first five IMFs, obtained after the EMD algorithm. It is evident from Plots 7 to 11 that as the order of the IMFs increases, the frequency content decreases. It is shown that the spectra of the first three IMFs correspond to the frequency band of the QRS complex. The dominant frequencies in Plots 7 to 9 (Fig. 9) are about 10-20 Hertz, whereas the dominant frequencies in Plots 10 and 11 are about 2-5 Hertz, which shows that the last two IMFs correspond to $\mathrm{P}$ and $\mathrm{T}$ waves, hence they should be discarded before signal reconstruction. Fig. 10 shows that the filtered signal, $x_{f}(t)$, can be approximated by the reconstructed signal, $x_{r}(t)$, because the difference of the two signals (yellow dotted line) is small and the shape of the QRS complex is preserved. Therefore, the first three IMFs are sufficient to designate the QRS complex. Our assumption was validated for all the recordings of Capnobase dataset, and the first three IMFs were found to be sufficient for reconstructing the signal, amplifying the QRS complex and reducing low frequency interference.

For all the recordings from Capnobase dataset the results obtained from our proposed QRS detector are shown in Table II. Table III shows a comparison of our method's performance with other existing detectors. Fig. 11 shows the sequential steps of the R-peak detection method. The detected R-peaks are marked by an asterisk ' ${ }^{*}$ ' on the filtered signal, $x_{f}(t)$ (Plot 4). A false negative (FN) occurs when the algorithm fails to detect an actual R-peak. A false positive (FP) represents a false peak detection. Sensitivity (Se), Positive Predictivity $(+\mathrm{P})$ and Detection Error Rate (DER) were calculated for each recordings using the following formulas respectively:

$$
\begin{gathered}
\operatorname{Se}(\%)=\frac{T P}{T P+F N} \%, \\
+P(\%)=\frac{T P}{T P+F P} \%, \\
D E R(\%)=\frac{F P+F N}{\text { total number of R peaks }} \%,
\end{gathered}
$$

where TP (true positives) is the total number of R-peaks correctly identified.

As can be seen from Table II and Table III our QRS detector shows a better performance for the Capnobase records, achieving a $\mathrm{Se}$ of $100 \%$, a higher $+P$ of $99.80 \%$ compared to $99.78 \%$ in [7] and $99.70 \%$ in [11], as well as a lower DER of $0.24 \%$ compared to $0.25 \%$ in [7] and $0.31 \%$ in [11].

During our experiments the following observations were found, compared to existing methods. Firstly, an important observation, which yields high detection error, is that the absolute amplitude of a Q-peak is larger than the R-peak. This was found to identify the Q-peak as a real R-peak, because the threshold is applied to the absolute of the reconstructed signal. To overcome this issue the first derivative of the ECG signal is computed. The derivative after an R-peak is negative because the signal decreases in time. The derivative after a Q-peak is positive as the signal increases in time. Hence, peaks with a negative derivative were investigated further in the decision stage by applying the refractory period check of 200 milliseconds. Secondly, another significant advantage of our proposed method is that it can be implemented in real-time with a detection delay equal to the duration of the 

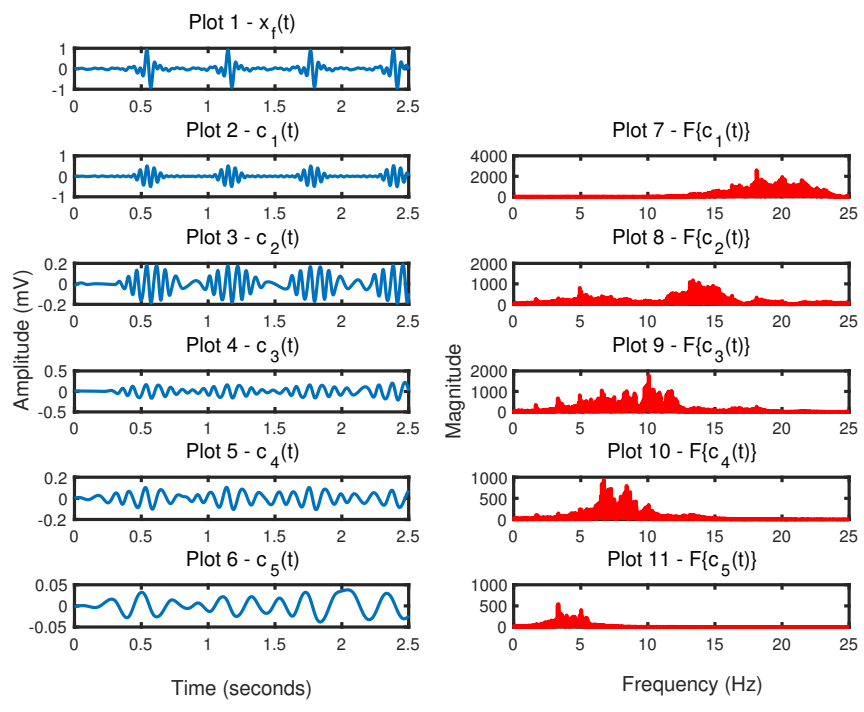

Fig. 9. The result on the EMD and the spectrum of each IMF. Plot 1 corresponds to the filtered ECG, $x_{f}(t)$. Plots 2 to 6 correspond to the first five IMFs. Plot 7 to 11 correspond to the Fourier transform of each IMF.

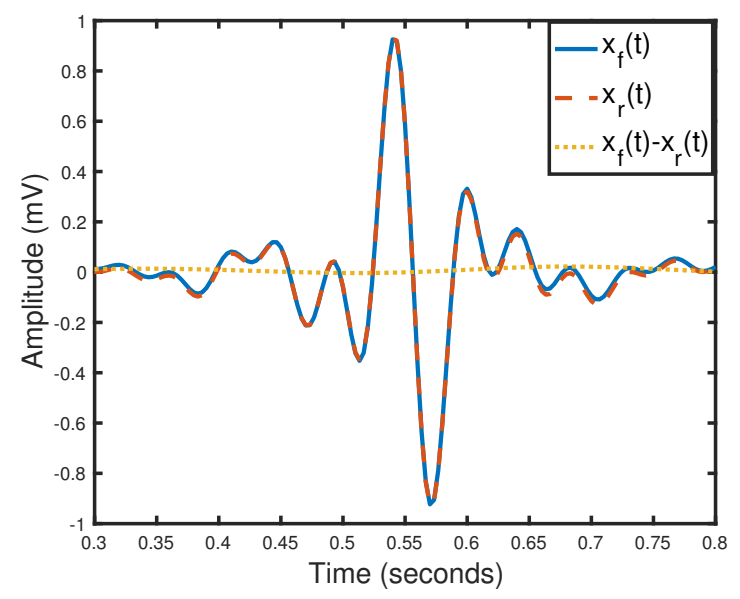

Fig. 10. Reconstruction of the filtered signal, $x_{f}(t)$, by the summation of the first three IMFs, $x_{r}(t)$, and their difference, $x_{f}(t)-x_{r}(t)$.

segment. On-line implementation requires a small alteration of our method. The segmentation can be executed at the very beginning of the pre-processing stage and the sequential steps of our algorithm can be implemented for each segment. Hence, the time between the task request and the availability of the detected R-peaks requires only that we await the part of the ECG signal of one segment time duration. For Capnobase recordings, for example, a segment of 3 seconds duration contains an adequate number of QRS complexes.

\section{CONCLUSION}

To conclude, a new ECG-Derived (EDR) respiration method was presented based on the accurate R-peak detection and the frequency domain analysis of the respiration in order to

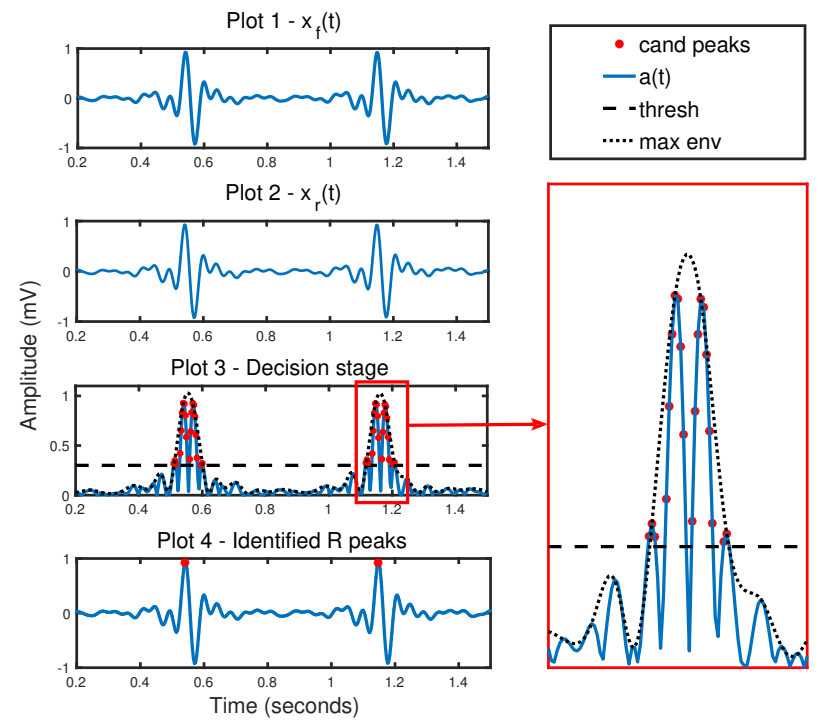

Fig. 11. Steps of the QRS detector for a part of the record 100 from the MITBIH database. Plot 1, corresponds to the filtered ECG signal, $x_{f}(t)$. Plot 2, corresponds to the reconstructed signal, $x_{r}(t)$. Plot 3 , shows the absolute sequence, $a_{k}(t)$, (blue line) and its maximum envelope, $\hat{a}_{k}(t)$, (dotted black line) along with the threshold (dashed black horizontal line) and candidate peaks marked with a red asterisk ' $*$ '. Plot 4 , shows the identified $\mathrm{R}$ peaks on $x_{f}(t)$ as red asterisk '*'.

estimate respiration rate. Our EDR method shows promising results for both real children and adult recordings from Capnobase dataset, achieving an average mean absolute error of 0.4150 breaths per minute, compared to 6.6987 of the time domain analysis [6]. The latter is important because no change of parameters was attempted during our experiments between children and adults. The novelty of our method results from the assumption that the dominant peak of the frequency spectrum of the respiration signal can give us an accurate estimation of the respiration rate. Additionally, a significant advantage of the proposed EDR method is that it can be executed in real-time with a detection delay of one minute.

Moreover, the present research suggested a new QRS detector based on the Empirical Mode Decomposition (EMD) using an adaptive threshold which relies on the local signal energy. This method provides a solution for the detection of small Rpeaks by establishing a threshold derived from the mean of the Root Mean Square over eight segments. Although our detector is based on EMD, it uses a gradient-based and refractory period checks to differentiate large Q-peaks and omit false R-peaks. Using the Capnobase dataset, the method performed effectively with accurate QRS complex detection of $99.80 \%$. Furthermore, the proposed QRS detector shows comparable results to other existing detectors using derivative-based [8] and Hilbert methods [12] on real data. In addition, the present method provides a real-time algorithm with a computational delay equal to the duration of the segment, whereas proposed methods require the full length ECG [16] [17]. 
TABLE II

QRS DETECTION PERFORMANCE USING THE CAPNOBASE DATASET

\begin{tabular}{|lllll|}
\hline Rec & Annotated peaks & DER $(\%)$ & $\boldsymbol{S e}(\%)$ & $\boldsymbol{+ P}(\%)$ \\
\hline 9 & 815 & 0.00 & 100 & 100 \\
15 & 960 & 0.00 & 100 & 100 \\
16 & 1012 & 0.00 & 100 & 100 \\
18 & 1131 & 0.00 & 100 & 100 \\
23 & 818 & 0.00 & 100 & 100 \\
32 & 685 & 0.00 & 100 & 100 \\
35 & 900 & 0.18 & 100 & 99.89 \\
38 & 956 & 0.00 & 100 & 100 \\
103 & 826 & 0.00 & 100 & 100 \\
104 & 912 & 0.00 & 100 & 100 \\
105 & 530 & 0.37 & 100 & 99.62 \\
121 & 579 & 0.00 & 100 & 100 \\
122 & 588 & 0.00 & 100 & 100 \\
125 & 627 & 0.00 & 100 & 100 \\
127 & 615 & 0.00 & 100 & 100 \\
128 & 541 & 0.18 & 100 & 99.82 \\
134 & 578 & 1.53 & 100 & 98.50 \\
142 & 739 & 0.00 & 100 & 100 \\
147 & 538 & 3.58 & 100 & 97.52 \\
148 & 624 & 0.00 & 100 & 100 \\
311 & 555 & 0.17 & 100 & 99.82 \\
312 & 432 & 0.00 & 100 & 100 \\
313 & 588 & 0.00 & 100 & 100 \\
322 & 589 & 0.16 & 100 & 99.83 \\
325 & 584 & 0.00 & 100 & 100 \\
\hline \hline Average $\mathbf{1 7 7 1 6}$ & $\mathbf{0 . 2 4}$ & $\mathbf{1 0 0}$ & $\mathbf{9 9 . 8 0}$ \\
\hline
\end{tabular}

TABLE III

COMPARISON OF QRS DETECTOR PERFORMANCE WITH OTHER METHODS FOR CAPNOBASE DATASET

\begin{tabular}{|cccc|}
\hline Method & DER (\%) & $\boldsymbol{S e}(\%)$ & $\boldsymbol{+ P}(\%)$ \\
\hline Derivative based [7] & 0.25 & 100 & 99.78 \\
Hilbert transform [11] & 0.31 & 100 & 99.70 \\
Our method & $\mathbf{0 . 2 4}$ & $\mathbf{1 0 0}$ & $\mathbf{9 9 . 8 0}$ \\
\hline
\end{tabular}

\section{REFERENCES}

[1] J. F Fieselmann, M. S. Hendryx, C. M Helms, and D. S. Wakefield, "Respiratory rate predicts cardiopulmonary arrest for internal medicine inpatients," Journal of General Internal medicine, vol. 8, pp. 354-360, 1993.

[2] D. R. Goldhill, A. F. McNarry, G. Mandersloot, and A. McGinley, "A physiologically-based early warning score for ward patients: the association between score and outcome," Anaesthesia, vol. 6, pp. 547$553,2005$.

[3] G. B. Moody, R. G. Mark, A. Zoccola, and S. Mantero, "Derivation of respiratory signals from multi-leas ECGs," Computers in Cardiology, vol. 12, pp. 113-116, 1985.

[4] E. Helfenbein, R. Firrozabadi, S. Chien, E. Carlson, and S. Babaeizadeh, "Development of three methods for extracting respiration from the surface ECG: a review," Journal of Electrocardiography, vol. 47, pp. 819-825, 2014.

[5] S. Babaizadeh, S. Zhou, S. D. Stephen, and D. P. White, "Electrocardiogram-derived respiration in screening of sleep-disordered breathing," Journal of Electrocardiology, vol. 44, pp. 700-706, 2011.

[6] S. A. Shah, "Vital sign monitoring and data fusion for paediatric triage," PhD Thesis, University of Oxford, 2012.

[7] G. B. Moody, "Spectral analysis of Heart Rate without resampling," in Computers in Cardiology, Proceedings, pp. 715-718, 1993.

[8] J. Pan, and W. J. Tompkins, "A real-time QRS detection algorithm," IEEE transactions on Biomedical Engineering, vol. 3, pp. 230-236, 1985.

[9] P. S. Hamilton, and W. J. Tompkins, "Quantitative investigation of QRS detection rules using the MIT/BIH Arrhythmia database," IEEE transactions on Biomedical Engineering, vol 12, pp. 1157-1165, 1986.
[10] N. Maglaveras, N. Stamkopoulos, T. Diamantaras, K. Pappas, and M. Strintzis, "ECG pattern recognition and classification using non-linear transformations and neural networks: A review," in International journal of Medical Informatics, vol. 52(1), pp.191-208, 1998.

[11] B. U. Kohler, C. Hennig, and R. Orglmeister, "The principles of software QRS detection," IEEE Engineering in Medicine and Biology Magazine, vol. 21(1), pp. 42-57, 2002.

[12] D. S. Benitez, P. A. Gaydecki, A. Zaidi, and A. P. Fitzpatrick, "A new QRS detection algorithm based on the Hilbert transform," in Computers in Cardiology, vol. 27, pp. 379-382, 2000.

[13] D. S. Benitez, P. A. Gaydecki, A. Zaidi, and A. P. Fitzpatrick, "The use of Hilbert transform in ECG signal analysis," Computers in Cardiology and Medicine, vol. 31(5), pp. 399-406, 2001.

[14] N. E. Huang et al., "The Empirical Mode Decomposition and the Hilbert spectrum for nonlinear and non-stationary time series analysis," Proceedings of the Royal Society of London A: Mathematical, Physical and Engineering Sciences, vol. 454, pp. 903-995, 1998.

[15] X. L. Yang, and J. T. Tang, "Hilbert-Huang transform and Wavelet transform for ECG detection," 4th International Conference on Wireless Communications, Networking and Mobile Computing, WiCOM'08, pp. $1-4,2008$.

[16] M. A. Arafat, and M. K. Hasan, "Automatic detection of ECG wave boundaries using Empirical Mode Decomposition," in ICASSP, IEEE International Conference on Acoustics, Speech and Signal Processing, pp. 461-466, 2009.

[17] S. Taouli, and F. Bereksi-Reguig, "Detection of QRS complexes in ECG signals based on Empirical Mode Decomposition," in Global Journal of Computer Science and Technology, vol. 11(20), 2011.

[18] J. Malmivuo, and R. Plonsey, Bioelectromagnetism: Principles and Applications of Bioelectric and Biomagnetic fields, Oxford University Press, 1995.

[19] J. G. Proakis, and D. G. Manolakis, "Digital Signal Processing: principles, algorithms, and applications (3rd edition)", Prentice hall, 1996.

[20] M. Elgendi, M. Jonkman, and F. DeBoer, "Frequency bands effects on QRS detection," in 3rd International Joint Conference on Biomedical Engineering Systems and Technologies (BIOSIGNALS), vol. 5, pp. 428431, 2010.

[21] W. Karlen, S. Raman, J. M. Ansermino, and G. A. Dumont, "Multiparameter respiratory rate estimation from photoplethysmogram," IEEE Transactions on Bio-medical Engineering, vol. 60(7), pp. 1946-1953, 2013. 